\title{
Solid hulls of weighted Banach spaces of entire functions
}

\author{
José Bonet and Jari Taskinen
}

\begin{abstract}
Given a continuous, radial, rapidly decreasing weight $v$ on the complex plane, we study the solid hull of its associated weighted space $H_{v}^{\infty}(\mathbb{C})$ of all the entire functions $f$ such that $v|f|$ is bounded. The solid hull is found for a large class of weights satisfying the condition (B) of Lusky. Precise formulations are obtained for weights of the form $v(r)=$ $\exp \left(-a r^{p}\right), a>0, p>0$. Applications to spaces of multipliers are included.
\end{abstract}

\section{Introduction and first results}

The aim of this paper is to investigate the solid hull of weighted Banach spaces $H_{v}^{\infty}(\mathbb{C})$ of all entire functions $f$ such that $\|f\|_{v}:=\sup _{z \in \mathbb{C}} v(z)|f(z)|$ is finite. In what follows, we identify an entire function $f(z)=\sum_{n=0}^{\infty} a_{n} z^{n}$ with the sequence of its Taylor coefficients $\left(a_{n}\right)_{n=0}^{\infty}$. For example in the case $v(z)=e^{-|z|}, z \in \mathbb{C}$, we show in Theorem 3.1 that the solid hull consists precisely of complex sequences $\left(b_{m}\right)_{m=0}^{\infty}$ such that

$$
\sup _{n \in \mathbb{N}} \sum_{m=n^{2}+1}^{(n+1)^{2}}\left|b_{m}\right|^{2} e^{-2 n^{2}} n^{4 m}<\infty .
$$

We are also able to characterize in Theorem 2.5, the solid hulls for a quite general class of weights in terms of numerical sequences defined by Lusky, [18], in his investigations of the isomorphic classes of the spaces $H_{v}^{\infty}(\mathbb{C})$. This class of weights includes those satisfying condition (B) of [18], see Remark 2.7 and Corollary 2.8. The calculation of the numerical sequences for some important weights $v$ is one of the results of our paper, see Proposition 3.2. In addition to techniques of [18], our approach uses the methods of Bennett, Stegenga and Timoney in their paper [2], where the solid hull and the solid core of the weighted spaces $H_{v}^{\infty}(\mathbb{D})$ were determined for doubling weights $v$ on the open unit disc $\mathbb{D}$. In Section 4 we show that

Mathematics Subject Classification (2010): Primary 46E15; Secondary 30D15, 30H20, 46B45, $46 \mathrm{E} 05$.

Keywords: Weighted Banach spaces of entire functions, Taylor coefficients, solid hull, solid core. 
our results in Section 2 can be used to determine space of multipliers from $H_{v}^{\infty}(\mathbb{C})$ into $\ell^{p}, 1 \leq p \leq \infty$.

The solid hull and multipliers on spaces of analytic functions on the disc has been investigated by many authors. In addition to [2], we mention here a non exhaustive sample: [1], [5], [11], [12], [13], [21] and the list of references in [7]. Moreover, the papers [6], [16] and [20] investigate the behavior of the Taylor coefficients of entire functions belonging to weighted spaces similar to those considered in this paper. Spaces of type $H_{v}^{\infty}(\mathbb{C})$ and $H_{v}^{\infty}(\mathbb{D})$ appear in the study of growth conditions of analytic functions and have been investigated in various articles since the work of Shields and Williams, see e.g. [3], [4], [9], [10], [17], [18], [19] and the references therein.

A weight $v$ is a continuous function $v:[0, \infty[\rightarrow] 0, \infty[$, which is non-increasing on $\left[0, \infty\left[\right.\right.$ and it is rapidly decreasing, i.e., it satisfies $\lim _{r \rightarrow \infty} r^{m} v(r)=0$ for each $m \in \mathbb{N}$. We extend $v$ to $\mathbb{C}$ by $v(z):=v(|z|)$. For such a weight, the weighted Banach space of entire functions is defined by

$$
H_{v}^{\infty}(\mathbb{C}):=\left\{f \in H(\mathbb{C}):\|f\|_{v}:=\sup _{z \in \mathbb{C}} v(|z|)|f(z)|<\infty\right\},
$$

and it is endowed with the weighted sup norm $\|\cdot\|_{v}$. Spaces of this type are also called sometimes weighted Fock spaces of infinite order. For an entire function $f \in H(\mathbb{C})$, we denote $M(f, r):=\max \{|f(z)|:|z|=r\}$. Using the notation $O$ and $o$ of Landau, $f \in H_{v}^{\infty}(\mathbb{C})$ if and only if $M(f, r)=O(1 / v(r)), r \rightarrow \infty$. The symbol $\mathbb{N}$ stands for the natural numbers $n=1,2,3, \ldots$.

As we already mentioned, an entire function $f(z)=\sum_{n=0}^{\infty} a_{n} z^{n}$ is identified with the sequence of its Taylor coefficients $\left(a_{n}\right)_{n=0}^{\infty}$, that will be also denoted sometimes by $\left(a_{n}\right)_{n}$. As is well known, it is often impossible to characterize standard Banach spaces of entire functions in terms of the Taylor coefficients; this is for example true for the function spaces $H_{v}^{\infty}(\mathbb{C})$. The next best thing is to find the strongest growth condition that the coefficients have to satisfy. This motivates the concept of a solid hull, and we now recall the related definitions from [1].

Let $A$ and $B$ be vector spaces of complex sequences containing the space of all the sequences with finitely many non-zero coordinates. The space $A$ is solid if $a=\left(a_{n}\right) \in A$ and $\left|b_{n}\right| \leq\left|a_{n}\right|$ for each $n$ implies $b=\left(b_{n}\right) \in A$. The solid hull of $A$ is

$$
S(A):=\left\{\left(c_{n}\right): \exists\left(a_{n}\right) \in A \text { such that }\left|c_{n}\right| \leq\left|a_{n}\right| \forall n \in \mathbb{N}\right\} .
$$

The solid core of $A$ is

$$
s(A):=\left\{\left(c_{n}\right):\left(c_{n} a_{n}\right) \in A \forall\left(a_{n}\right) \in \ell_{\infty}\right\} .
$$

The set of multipliers form $A$ into $B$ is

$$
(A, B):=\left\{c=\left(c_{n}\right):\left(c_{n} a_{n}\right) \in B \forall\left(a_{n}\right) \in A\right\} .
$$

We conclude this section with our first results.

Proposition 1.1. The solid core of $H_{v}^{\infty}(\mathbb{C})$ is

$$
s(v, \mathbb{C}):=\left\{\left(a_{n}\right)_{n}:\left\|\left(a_{n}\right)\right\|_{1, v, \mathbb{C}}:=\sup _{r>0} v(r) \sum_{n=0}^{\infty}\left|a_{n}\right| r^{n}<\infty\right\} .
$$


Proof. Given $\left(a_{n}\right)_{n} \in s(v, \mathbb{C})$, the function $f(z)=\sum_{n=0}^{\infty} a_{n} z^{n}$ is clearly entire. Moreover $v(|z|)|f(z)| \leq\left\|\left(a_{n}\right)_{n}\right\|_{1, v, \mathbb{C}}$ for each $z \in \mathbb{C}$, and $f \in H_{v}^{\infty}(\mathbb{C})$.

To see the other inclusion, let $A$ be a solid sequence space contained in $H_{v}^{\infty}(\mathbb{C})$, i.e., for each $\left(a_{n}\right)_{n} \in A,\left(\left|a_{n}\right|\right)_{n} \in A$ and $g(z)=\sum_{n=0}^{\infty}\left|a_{n}\right| z^{n} \in H_{v}^{\infty}(\mathbb{C})$. Clearly,

$$
M(g, r)=\sup _{|z|=r}\left|\sum_{n=0}^{\infty}\right| a_{n}\left|z^{n}\right|=\sum_{n=0}^{\infty}\left|a_{n}\right| r^{n} .
$$

Therefore,

$$
\sup _{r>0} v(r) \sum_{n=0}^{\infty}\left|a_{n}\right| r^{n}=\sup _{r>0} v(r) M(g, r)<\infty .
$$

Remark 1.2. Write for $n \in \mathbb{N},\left\|z^{n}\right\|_{v}:=\sup _{r>0} v(r) r^{n}$. Clearly $\left\|z^{0}\right\|_{v}=v(0)$. The weighted $\ell_{1}$ space $\ell_{1}\left(\left(\left\|z^{n}\right\|_{v}\right)\right.$ of all those complex sequences $\left(a_{n}\right)_{n}$ such that $\sum_{n=0}^{\infty}\left|a_{n}\right|\left\|z^{n}\right\|_{v}<\infty$ is contained in the solid core $s(v, \mathbb{C})$. However, in general the inclusion is strict. Indeed, if $v(r)=e^{-r}, r \geq 0$, then $\left\|z^{n}\right\|_{v}=(n / e)^{n}$ as a direct calculation shows. Take $a_{n}:=1 / n !, n=0,1,2, \ldots$ For each $r>0$, we have $v(r) \sum_{n=0}^{\infty} a_{n} r^{n}=1$. However, the series $\sum_{n=0}^{\infty}\left(n^{n} / n ! e^{n}\right)$ diverges by the Stirling's formula $n ! \sim(2 \pi n)^{1 / 2}(n / e)^{n}$.

Our next elementary result about the behavior of the Taylor coefficients of elements $f \in H_{v}^{\infty}(\mathbb{C})$, that holds for arbitrary weights $v$, clarifies the importance of the study of the solid hull of $H_{v}^{\infty}(\mathbb{C})$ in Section 2 .

Proposition 1.3. The Taylor coefficients of an entire function $f(z)=\sum_{n=0}^{\infty} a_{n} z^{n}$ in $H_{v}^{\infty}(\mathbb{C})$ satisfy

(i) $\sup _{r>0} v(r)\left(\sum_{n=0}^{\infty}\left|a_{n}\right|^{2} r^{2 n}\right)^{1 / 2} \leq\|f\|_{v}$, and

(ii) $\sup _{n}\left|a_{n}\right|\left\|z^{n}\right\|_{v} \leq\|f\|_{v}$.

Proof. (i) Since $H^{\infty}$ is contained in $H^{2}$, for each function $h(z)=\sum_{n=0}^{\infty} b_{n} z^{n}$ analytic in a neighbourhood of $\mathbb{D}$, we have $\left(\sum_{n=0}^{\infty}\left|b_{n}\right|^{2}\right)^{1 / 2} \leq M(h, 1)$. Now, given $f(z)=\sum_{n=0}^{\infty} a_{n} z^{n} \in H_{v}^{\infty}(\mathbb{C})$ and $r>0$, set $g(\zeta):=f(r \zeta)$, that is clearly an analytic function in a neighbourhood of $\mathbb{D}$. Since $g(\zeta)=f(r \zeta)=\sum_{n=0}^{\infty} a_{n} r^{n} \zeta^{n}$, we conclude

$$
\left(\sum_{n=0}^{\infty}\left|a_{n}\right|^{2} r^{2 n}\right)^{1 / 2} \leq M(g, 1)=M(f, r) .
$$

This implies the inequality in the statement (i).

(ii) follows from (i).

Remark 1.4. In general it is not true that $f \in H_{v}^{\infty}(\mathbb{C})$ implies that $\left(\left|a_{n}\right|\left\|z^{n}\right\|_{v}\right)_{n} \in \ell_{2}$. To see this, take again $v(r)=e^{-r}, r \geq 0$. Clearly $e^{z}=\sum_{n=0}^{\infty}(1 / n !) z^{n}$ belongs to $H_{v}^{\infty}(\mathbb{C})$. However, the series $\sum_{n=0}^{\infty}\left(\left|\bar{a}_{n}\right|\left\|z^{n}\right\|_{v}\right)^{2}=\sum_{n=0}^{\infty}\left(n^{n} / n ! e^{n}\right)^{2}$ diverges because $\left(n^{n} / n ! e^{n}\right)^{2} \sim 1 / n$ by Stirling's formula. 


\section{The solid hull of $H_{v}^{\infty}(\mathbb{C})$}

We fix for this section a weight $v: \mathbb{C} \rightarrow] 0, \infty[$ satisfying the general hypothesis made in Section 1. Our next aim is to characterize the solid hull of $H_{v}^{\infty}(\mathbb{C})$ for weights satisfying the additional condition (2.2), below. Let us start by introducing some notation used in [18].

We denote by $[x]$ be the largest integer less or equal $x$ for a given real number $x \in \mathbb{R}$. Given $m>0$, we denote by $r_{m}$ the global maximum point of $r^{m} v(r)$. Then $r_{m} \rightarrow \infty$ as $m \rightarrow \infty$. For example, if $v(r)=\exp \left(-\alpha r^{p}\right)$, then $r_{m}=$ $(m / \alpha p)^{1 / p}, m>0$. Given an entire function $f(z)=\sum_{k=0}^{\infty} a_{k} z^{k}$, and $0<m<n$ (not necessarily integers) we define the following operators of de la Vallée-Poussin type:

$$
\begin{aligned}
V_{n, m} f & :=\sum_{0 \leq k \leq m} a_{k} z^{k}+\sum_{m<k \leq n} \frac{[n]-k}{[n]-[m]} a_{k} z^{k}, \\
V_{p, 0} f & :=\sum_{0 \leq k \leq p} \frac{[p]-k}{[p]} a_{k} z^{k}
\end{aligned}
$$

here and later, the summation is performed over integers belonging to the given intervals, although the endpoints of the intervals need not be integers. We also denote, for $0<m<n$,

$$
A(m, n):=\left(\frac{r_{m}}{r_{n}}\right)^{m} \frac{v\left(r_{m}\right)}{v\left(r_{n}\right)} \quad \text { and } \quad B(m, n):=\left(\frac{r_{n}}{r_{m}}\right)^{n} \frac{v\left(r_{n}\right)}{v\left(r_{m}\right)} .
$$

Several results of Lusky, [18], will be needed below. We start with the following lemma.

Lemma 2.1 ([18], Corollary $3.2(\mathrm{~b}))$. Let $0<m<n$ and let $Q(z)=\sum_{m<k \leq n} a_{k} z^{k}$ be a polynomial. Then

$$
\|Q\|_{v} \leq 2 A(m, n) \sup _{|z|=r_{n}}|Q(z)| v(z), \quad \text { and } \quad\|Q\|_{v} \leq 2 B(m, n) \sup _{|z|=r_{m}}|Q(z)| v(z) .
$$

Given a strictly increasing sequence $\left(m_{n}\right)_{n=1}^{\infty}$ with $\lim _{n \rightarrow \infty} m_{n}=\infty$, we define $m_{0}=0$ and

$$
V_{n}:=V_{m_{n+1}, m_{n}}-V_{m_{n}, m_{n-1}}, \quad n \in \mathbb{N} .
$$

For each $n \in \mathbb{N}, n \geq 2, V_{n} f$ is a polynomial with all terms of degree at least $m_{n-1}+1$ and at most $m_{n+1}$, for all $f \in H(\mathbb{C})$. In fact, for each $f(z)=\sum_{n=0}^{\infty} f_{n} z^{n} \in H(\mathbb{C})$, we have

$$
V_{n} f(z)=\sum_{m_{n-1}<m \leq m_{n+1}} \gamma_{m} f_{m} z^{m}=\sum_{m=\left[m_{n-1}\right]+1}^{\left[m_{n+1}\right]} \gamma_{m} f_{m} z^{m},
$$

where the numbers $\gamma_{m} \in[0,1]$ are

$$
\gamma_{m}=\frac{\left[m_{n+1}\right]-m}{\left[m_{n+1}\right]-\left[m_{n}\right]}, \quad m_{n}<m \leq m_{n+1}
$$


and

$$
\gamma_{m}=\frac{m-\left[m_{n-1}\right]}{\left[m_{n}\right]-\left[m_{n-1}\right]}, \quad m_{n-1}<m \leq m_{n} .
$$

The sum in $V_{1} f$ is understood to go from $m=0$ to $m=m_{1}$.

Lemma 2.2 (Proposition $3.4(\mathrm{~b})$ in [18]). If the sequence $\left(m_{n}\right)_{n}$, with $\lim _{n \rightarrow \infty} m_{n}$ $=\infty$, satisfies

$$
\min \left(A\left(m_{n}, m_{n+1}\right), B\left(m_{n}, m_{n+1}\right)\right) \geq b
$$

for some $b>2$, then there is $D>0$ such that $\left\|V_{n}\right\| \leq D$ for each $n \in \mathbb{N}$, where $\|\cdot\|$ is the operator norm in $L\left(H_{v}^{\infty}(\mathbb{C})\right)$ with respect to $\|\cdot\|_{v}$.

Lemma 2.3 (Lemma 5.1 in $[18]$ ). Fix $b>1$. For each weight $v:[0, \infty[\rightarrow] 0, \infty[$ there is a sequence of numbers $0<m_{1}<m_{2}<\cdots$, with $\lim _{n \rightarrow \infty} m_{n}=\infty$, such that

$$
A\left(m_{n}, m_{n+1}\right) \geq b \quad \text { and } \quad B\left(m_{n}, m_{n+1}\right) \geq b,
$$

and

$$
\sup _{n \in \mathbb{N}} \min \left(A\left(m_{n}, m_{n+1}\right), B\left(m_{n}, m_{n+1}\right)\right)<\infty .
$$

In fact, Lusky proves in Lemma 5.1 in [18] that $\left(m_{n}\right)_{n}$ can be selected to satisfy $\min \left(A\left(m_{n}, m_{n+1}\right), B\left(m_{n}, m_{n+1}\right)\right)=b$ for each $n \in \mathbb{N}$. Our next lemma is contained in Proposition 5.2 of [18], and its proof in [18] only uses the fact that $\min \left(A\left(m_{n}, m_{n+1}\right), B\left(m_{n}, m_{n+1}\right)\right) \geq b$, not that $\min \left(A\left(m_{n}, m_{n+1}\right), B\left(m_{n}, m_{n+1}\right)\right)$ $=b$ for each $n \in \mathbb{N}$. (In Remark 3.6 we will be able to clarify, which of these quantities $A$ or $B$ is larger in the case of the weights $\exp \left(-a r^{p}\right)$.) Accordingly, the following result holds true, and it contains an important expression for a norm equivalent to that of $H_{v}^{\infty}(\mathbb{C})$.

Lemma 2.4 (Proposition 5.2 in [18]). Let the numbers $\left(m_{n}\right)_{n=1}^{\infty}$ and $b$ be as in Lemma 2.3, and in addition assume that $b>2$. Then, for every $f \in H_{v}^{\infty}(\mathbb{C})$,

$$
c_{1} \sup _{n \in \mathbb{N} r_{m_{n-1}} \leq|z| \leq r_{m_{n+1}}} \sup _{n}\left|f_{n}(z)\right| v(z) \leq\|f\|_{v} \leq c_{2} \sup _{n \in \mathbb{N}} \sup _{r_{m_{n-1}} \leq|z| \leq r_{m_{n+1}}}\left|f_{n}(z)\right| v(z),
$$

where $f_{n}:=V_{n} f$.

The main result of this section reads as follows.

Theorem 2.5. Let $v: \mathbb{C} \rightarrow] 0, \infty[$ be a weight (which is continuous, radial, nonincreasing on $[0, \infty[$, and rapidly decreasing). In addition, assume that there exists a sequence $0<m_{1}<m_{2}<\cdots$, with $\lim _{n \rightarrow \infty} m_{n}=\infty$, such that for some $b>2$ and some $K \geq b$ we have

$$
\begin{aligned}
b & \leq \min \left(A\left(m_{n}, m_{n+1}\right), B\left(m_{n}, m_{n+1}\right)\right) \\
& \leq \max \left(A\left(m_{n}, m_{n+1}\right), B\left(m_{n}, m_{n+1}\right)\right) \leq K,
\end{aligned}
$$

for each $n \in \mathbb{N}$. 
Then, the solid hull of $H_{v}^{\infty}(\mathbb{C})$ is

$$
S(v, \mathbb{C}):=\left\{\left(b_{m}\right)_{m=0}^{\infty}: \sup _{n \in \mathbb{N}} v\left(r_{m_{n}}\right)\left(\sum_{m_{n}<m \leq m_{n+1}}\left|b_{m}\right|^{2} r_{m_{n}}^{2 m}\right)^{1 / 2}<\infty\right\}
$$

Remark 2.6. Notice that if a weight $v$, as in Theorem 2.5, satisfies (2.2), then the conclusion of Lemma 2.4 also holds. In fact, many important weights do satisfy (2.2), see Corollary 2.8 and Remark 2.7. Moreover, examples with explicit calculations of the sequence $\left(m_{n}\right)_{n}$ will be presented in Section 3 .

Of course, there is no need to extend the condition (2.3) to the finitely many coefficients $b_{m}, 0 \leq m \leq m_{1}$.

In the characterization (2.3) we could as well replace $r_{m_{n}}$ by $r_{m_{n+1}}$. This follows from the proof below, or as well from the the right-hand side inequality in the assumption (2.2) together with the definition (2.1). Namely, the condition (2.2) implies

$$
\frac{1}{K} \leq\left(\frac{r_{m_{n+1}}}{r_{m_{n}}}\right)^{m_{n}} \frac{v\left(r_{m_{n+1}}\right)}{v\left(r_{m_{n}}\right)} \leq\left(\frac{r_{m_{n+1}}}{r_{m_{n}}}\right)^{m_{n+1}} \frac{v\left(r_{m_{n+1}}\right)}{v\left(r_{m_{n}}\right)} \leq K
$$

Hence, for every $m$ with $m_{n} \leq m \leq m_{n+1}$ we have

$$
\frac{1}{K} \leq\left(\frac{r_{m_{n+1}}}{r_{m_{n}}}\right)^{m} \frac{v\left(r_{m_{n+1}}\right)}{v\left(r_{m_{n}}\right)} \leq K
$$

or, for all $m_{n} \leq m \leq m_{n+1}$,

$$
\frac{1}{K} r_{m_{n}}^{m} v\left(r_{m_{n}}\right) \leq r_{m_{n+1}}^{m} v\left(r_{m_{n+1}}\right) \leq K r_{m_{n}}^{m} v\left(r_{m_{n}}\right)
$$

This means that $r_{m_{n}}^{m} v\left(r_{m_{n}}\right)$ may be replaced by $r_{m_{n+1}}^{m} v\left(r_{m_{n+1}}\right)$ in $(2.3)$.

Proof. The proof will be obtained in two steps.

Step 1. If $g(z)=\sum_{m=0}^{\infty} b_{m} z^{m} \in H_{v}^{\infty}(\mathbb{C})$, and $\|g\|_{v} \leq 1$, then there is $C>0$ such that

$$
\left(\sum_{m_{n}<m \leq m_{n+1}}\left|b_{m}\right|^{2} r_{m_{n}}^{2 m}\right)^{1 / 2} \leq \frac{C}{v\left(r_{m_{n}}\right)}
$$

for all $n \in \mathbb{N}$.

This step is the analogue in our setting of Theorem 1.8 in [2].

We estimate first the sum from $m_{n}$ to $\left(m_{n}+m_{n+1}\right) / 2$. To do this, observe that 
if $m_{n} \leq m \leq\left(m_{n}+m_{n+1}\right) / 2$, then $\gamma_{m} \geq 1 / 2$. We have

$$
\begin{aligned}
\left(\sum_{m=\left[m_{n}\right]+1}^{\left[\left(m_{n}+m_{n+1}\right) / 2\right]}\left|b_{m}\right|^{2} r_{m_{n}}^{2 m}\right)^{1 / 2} \leq 2\left(\sum_{m=\left[m_{n}\right]+1}^{\left[\left(m_{n}+m_{n+1}\right) / 2\right]} \gamma_{m}^{2}\left|b_{m}\right|^{2} r_{m_{n}}^{2 m}\right)^{1 / 2} \\
\leq 2\left(\sum_{m=\left[m_{n-1}\right]+1}^{\left[m_{n+1}\right]} \gamma_{m}^{2}\left|b_{m}\right|^{2} r_{m_{n}}^{2 m}\right)^{1 / 2} \\
=2\left(\int_{\partial \mathbb{D}}\left|\sum_{m_{n-1}<m \leq m_{n+1}} \gamma_{m} b_{m} r_{m_{n}}^{m} z^{m}\right|^{2} d z\right)^{1 / 2} \\
\leq 4 \pi \sup _{|z|=1}\left|\sum_{m_{n-1}<m \leq m_{n+1}} \gamma_{m} b_{m} r_{m_{n}}^{m} z^{m}\right| \\
=4 \pi \sup _{|z|=r_{m_{n}} \mid}\left|\sum_{m_{n-1}<m \leq m_{n+1}} \gamma_{m} b_{m} r_{m_{n}}^{m}\left(\frac{z}{r_{m_{n}}}\right)^{m}\right| \\
=\frac{4 \pi}{v\left(r_{m_{n}}\right)} v\left(r_{m_{n}}\right) \sup _{|z|=r_{m_{n}}}\left|\sum_{m_{n-1}<m \leq m_{n+1}} \gamma_{m} b_{m} z^{m}\right| \\
=\frac{4 \pi}{v\left(r_{m_{n}}\right)} v\left(r_{m_{n}}\right) \sup _{|z|=r_{m_{n}}}\left|V_{n} g(z)\right| \leq \frac{4 \pi|| V_{n} g \|_{v}}{v\left(r_{m_{n}}\right)} \leq \frac{4 \pi D}{v\left(r_{m_{n}}\right)}
\end{aligned}
$$

since the operators $V_{n}$ are uniformly bounded with respect to $\|\cdot\|_{v}$ by $D>0$, by Lemma 2.2 and (2.2).

Now we estimate the sum from $\left(m_{n}+m_{n+1}\right) / 2$ to $m_{n+1}$. Observe that

$$
V_{n+1} g(z)=\sum_{m_{n}<m \leq m_{n+2}} \tilde{\gamma}_{m} b_{m} z^{m}=\sum_{m=\left[m_{n}\right]+1}^{\left[m_{n+2}\right]} \tilde{\gamma}_{m} b_{m} z^{m}
$$

where the numbers $\tilde{\gamma}_{m} \in[0,1]$ are

$$
\tilde{\gamma}_{m}=\frac{m-\left[m_{n}\right]}{\left[m_{n+1}\right]-\left[m_{n}\right]}, \quad m_{n}<m \leq m_{n+1},
$$

which increase from $\tilde{\gamma}_{m_{n}+1}$ till $\tilde{\gamma}_{m_{n+1}}=1$. If $\left(m_{n}+m_{n+1}\right) / 2<m \leq m_{n+1}$, we have $\tilde{\gamma}_{m} \geq 1 / 2$. Thus, proceeding similarly as we did before, we get

$$
\begin{aligned}
& \left(\sum_{m=\left[\left(m_{n}+m_{n+1}\right) / 2\right]+1}^{\left[m_{n+1}\right]}\left|b_{m}\right|^{2} r_{m_{n}}^{2 m}\right)^{1 / 2} \leq 2\left(\sum_{m=\left[\left(m_{n}+m_{n+1}\right) / 2\right]+1}^{\left[m_{n+1}\right]} \tilde{\gamma}_{m}^{2}\left|b_{m}\right|^{2} r_{m_{n}}^{2 m}\right)^{1 / 2} \\
& \quad \leq 2\left(\sum_{m=\left[m_{n}\right]+1}^{\left[m_{n+2}\right]} \tilde{\gamma}_{m}^{2}\left|b_{m}\right|^{2} r_{m_{n}}^{2 m}\right)^{1 / 2} \leq \frac{4 \pi}{v\left(r_{m_{n}}\right)} v\left(r_{m_{n}}\right) \sup _{|z|=r_{m_{n}}}\left|V_{n+1} g(z)\right| \\
& \quad \leq \frac{4 \pi\left\|V_{n+1} g\right\|_{v}}{v\left(r_{m_{n}}\right)} \leq \frac{4 \pi D}{v\left(r_{m_{n}}\right)}
\end{aligned}
$$

This completes the proof of Step 1. 
Observe that the estimates proved in Step 1 remain valid if we replace $r_{m_{n}}$ by any $r_{m}$ with $m_{n} \leq m \leq m_{n+1}$.

Step 2. For each $\left(b_{m}\right)_{m=0}^{\infty} \in S(v, \mathbb{C})$ there is $f(z)=\sum_{m=0}^{\infty} a_{m} z^{m} \in H_{v}^{\infty}(\mathbb{C})$ such that $\left|b_{m}\right| \leq\left|a_{m}\right|$ for each $m=0,1,2, \ldots$.

Fix $C_{0}>\sup _{n} v\left(r_{m_{n}}\right)\left(\sum_{m_{n}<m \leq m_{n+1}}\left|b_{m}\right|^{2} r_{m_{n}}^{2 m}\right)^{1 / 2}$.

For all $n \in \mathbb{N}$ and $m_{n}<m \leq m_{n+1}$, we apply Corollary 2.8 in [2] (which is a consequence of a deep result of Kisliakov [15]), to the sequence

$$
\left(b_{m} r_{m_{n}}^{m}\right)_{m_{n}<m \leq m_{n+1}},
$$

to choose a polynomial

$$
P_{n}(z)=\sum_{m_{n}<m \leq m_{n+1}} b_{m}^{\prime} z^{m}
$$

such that

$$
\begin{gathered}
\left|b_{m}^{\prime}\right| \geq\left|b_{m}\right| r_{m_{n}}^{m} \quad \forall m, \text { and } \\
\sup _{|z|<1}\left|P_{n}(z)\right|=\sup _{|z|=1}\left|P_{n}(z)\right| \leq B\left(\sum_{m_{n}<m \leq m_{n+1}}\left|b_{m}\right|^{2} r_{m_{n}}^{2 m}\right)^{1 / 2} .
\end{gathered}
$$

Here $B>0$ is an absolute constant. Define

$$
Q_{n}(z):=\sum_{m_{n}<m \leq m_{n+1}} r_{m_{n}}^{-m} b_{m}^{\prime} z^{m}
$$

and

$$
g(z)=\sum_{n=1}^{\infty} Q_{n}(z)=\sum_{n=1}^{\infty} \sum_{m_{n}<m \leq m_{n+1}} r_{m_{n}}^{-m} b_{m}^{\prime} z^{m} .
$$

We still have to show that $g$ is a well defined entire function and that $g \in H_{v}^{\infty}(\mathbb{C})$. However, observe that if $a_{m}$ denotes the $m$-th Taylor coefficient of $g$, then $\left|a_{m}\right| \geq$ $\left|b_{m}\right|$ for all $m$.

By Lemma 2.1, (2.2) and (2.9), we have, for each $n \in \mathbb{N}$,

$$
\begin{aligned}
& \left\|Q_{n}\right\|_{v} \leq 2 K \sup _{|z|=m_{m_{n}}}\left|Q_{n}(z)\right| v(z)=2 K \sup _{|z|=m_{m}}\left|\sum_{m_{n}<m \leq m_{n+1}} r_{m_{n}}^{-m} b_{m}^{\prime} z^{m}\right| v(z) \\
& =2 K \sup _{|z|=r_{m_{n}}}\left|\sum_{m_{n}<m \leq m_{n+1}} b_{m}^{\prime}\left(z / r_{m_{n}}\right)^{m}\right| v(z)=2 K v\left(r_{m_{n}}\right) \sup _{|z|=1}\left|\sum_{m_{n}<m \leq m_{n+1}} b_{m}^{\prime} z^{m}\right| \\
& =2 K v\left(r_{m_{n}}\right) \sup _{|z|<1}\left|P_{n}(z)\right| \\
& \leq 2 K B v\left(r_{m_{n}}\right)\left(\sum_{m_{n}<m \leq m_{n+1}} r_{m_{n}}^{2 m}\left|b_{m}\right|^{2}\right)^{1 / 2} \leq 2 K B C_{0},
\end{aligned}
$$


Moreover, for all $n \geq 2$, we have

$$
\begin{aligned}
& \sup _{r_{m_{n-1}} \leq|z| \leq r_{m_{n+1}}}\left|V_{n}\left(Q_{n}+Q_{n-1}\right)(z)\right| v(z) \leq \sup _{z \in \mathbb{C}}\left|V_{n}\left(Q_{n}+Q_{n-1}\right)(z)\right| v(z) \\
& \leq D \sup _{z \in \mathbb{C}}\left|\left(Q_{n}+Q_{n-1}\right)(z)\right| v(z) \leq D\left\|Q_{n}\right\|_{v}+D\left\|Q_{n-1}\right\|_{v} \leq 4 K B C_{0} D .
\end{aligned}
$$

Consequently, for each $N>n \geq 2$, we have

$$
V_{n}\left(\sum_{j=2}^{N} Q_{j}\right)(z)=V_{n}\left(Q_{n}+Q_{n-1}\right)(z), \quad z \in \mathbb{C} .
$$

By Lemma 2.4 and (2.10) we get, for every $N \in \mathbb{N}$,

$$
\begin{aligned}
\left\|\sum_{j=2}^{N} Q_{j}\right\|_{v} & =\sup _{z \in \mathbb{C}} v(z)\left|\sum_{j=2}^{N} Q_{j}(z)\right| \leq c_{2} \sup _{n \in \mathbb{N}} \sup _{\substack{r_{m_{n}-1} \leq \\
|z| \leq r_{m}+1}}\left|V_{n}\left(\sum_{j=2}^{N} Q_{j}(z)\right)\right| v(z) \\
& =c_{2} \sup _{n \in \mathbb{N}} \sup _{\substack{r_{m_{n}-1} \leq \\
|z| \leq r_{m_{n+1}}}}\left|V_{n}\left(Q_{n}+Q_{n-1}\right)(z)\right| v(z) \leq 4 K B C_{0} c_{2} D .
\end{aligned}
$$

This implies that the sequence of polynomials $\left(\sum_{j=2}^{N} Q_{j}\right)_{N}$ is contained in a multiple of the unit ball of $H_{v}^{\infty}(\mathbb{C})$, which is compact for the compact open topology. Accordingly, there is a subsequence $\left(\sum_{j=2}^{N(s)} Q_{j}\right)_{s}$ converging to $h \in H_{v}^{\infty}(\mathbb{C})$ for this topology. Since the operator of $k$-th differentiation is continuous for the compact open topology, it follows that the Taylor coefficients of $h$ and $g-Q_{1}$ coincide. This implies that $g$ is an entire function and that $g \in H_{v}^{\infty}(\mathbb{C})$. The proof of Step 2 is now complete by taking the function $f(z):=g(z)+\sum_{0 \leq m \leq m_{1}} b_{m} z^{m}$.

Remark 2.7. (1) Lusky introduces the following condition (B) on the weight $v$ in [18]:

$$
\begin{gathered}
\forall b_{1}>0 \exists b_{2}>1 \exists c>0 \forall m, n: \\
\left(\frac{r_{m}}{r_{n}}\right)^{m} \frac{v\left(r_{m}\right)}{v\left(r_{n}\right)} \leq b_{1} \text { and }|m-n| \geq c \Rightarrow\left(\frac{r_{n}}{r_{m}}\right)^{n} \frac{v\left(r_{n}\right)}{v\left(r_{m}\right)} \leq b_{2} .
\end{gathered}
$$

By Theorem 1.1 in [18], if $v$ has condition $(\mathrm{B})$, then $H_{v}^{\infty}(\mathbb{C})$ is isomorphic to $\ell_{\infty}$, and if $v$ does not satisfy condition $(\mathrm{B})$, then $H_{v}^{\infty}(\mathbb{C})$ is isomorphic to $H^{\infty}$.

We show that if the weight $v$ satisfies condition (B), then for each $b>2$ one can find a sequence $\left(m_{n}\right)_{n}$ satisfying the assumption (2.2) in Theorem 2.5. Indeed, let $v$ be a weight satisfying condition (B). Given $b>2$ we apply Lemma 2.3 to find a sequence $0<m_{1}<m_{2}<\cdots$, with $\lim _{n \rightarrow \infty} m_{n}=\infty$, such that $A\left(m_{n}, m_{n+1}\right) \geq b$, $B\left(m_{n}, m_{n+1}\right) \geq b$ and $M:=\sup _{n} \min \left(A\left(m_{n}, m_{n+1}\right), B\left(m_{n}, m_{n+1}\right)\right)<\infty$. An inspection of the proof of Lemma 5.1 in Lusky shows that we can take in our Lemma 2.3 the sequence $\left(m_{n}\right)_{n}$ such that $\lim _{n \rightarrow \infty}\left(m_{n+1}-m_{n}\right)=\infty$. Set $b_{1}:=M$, and select $b_{2}>1$ and $c>0$ according to condition (B). There is $n(0) \in \mathbb{N}$ such that $m_{n+1}-m_{n} \geq c$ if $n \geq n(0)$. Condition (B) now implies that $\max \left(A\left(m_{n}, m_{n+1}\right)\right.$, $\left.B\left(m_{n}, m_{n+1}\right)\right) \leq \max \left(M, b_{2}\right)$ for each $n \geq n(0)$. The proof is complete if we take $\left(m_{n}\right)_{n \geq n(0)}$. 
(2) Lusky constructs in Example 2.6 in [18] a weight $v$ on $\mathbb{C}$ not satisfying condition (B) such that for a certain sequence $\left(m_{n}\right)$ with $m_{n+1}-m_{n}=n+1$, $A\left(m_{n}, m_{n+1}\right)=(n+1)^{n+1}$ and $B\left(m_{n}, m_{n+1}\right)=1$ for each $n \in \mathbb{N}$.

This remark and Examples 2.1-2.2 in [18] imply the following result.

Corollary 2.8. Condition (2.2) is satisfied by the following weights: $v(r)=$ $\exp \left(-r^{p}\right)$ with $p>0, v(r)=\exp (-\exp r)$, and $v(r)=\exp \left(-\left(\log ^{+} r\right)^{p}\right)$, where $p \geq 2$ and $\log ^{+} r=\max (\log r, 0)$.

\section{Examples}

In this section we calculate the sequences $\left(m_{n}\right)_{n=1}^{\infty}$ for the weights $v(r)=\exp \left(-a r^{p}\right)$ and thus obtain satisfactory representations of the corresponding solid hulls.

Theorem 3.1. Let $v$ be the weight $v(r)=\exp \left(-a r^{p}\right)$ on $\mathbb{C}$, where $a>0$ and $p>0$ are constants. Then, the solid hull of $H_{v}^{\infty}(\mathbb{C})$ is

$$
\left\{\left(b_{m}\right)_{m=0}^{\infty}: \sup _{n \in \mathbb{N}} \sum_{p n^{2}+1<m \leq p(n+1)^{2}}\left|b_{m}\right|^{2} e^{-2 n^{2}} n^{4 m / p}(a p)^{-m / p}<\infty\right\} .
$$

In particular, the solid hull for $v(r)=\exp (-r)$ is

$$
\left\{\left(b_{m}\right)_{m=0}^{\infty}: \sup _{n \in \mathbb{N}} \sum_{m=n^{2}+1}^{(n+1)^{2}}\left|b_{m}\right|^{2} e^{-2 n^{2}} n^{4 m}<\infty\right\} .
$$

Theorem 3.1 is an immediate consequence of Theorem 2.5 and the following proposition, where we choose $b=e$. The proposition gives the Lusky numbers $\left(m_{n}\right)_{n}$ for a class of important weights.

Proposition 3.2. Let $v(r)=\exp \left(-a r^{p}\right), a>0, p>0$ and let $b>2$. The sequence $m_{n}:=p(\log b) n^{2}, n \in \mathbb{N}$, satisfies, for each $n \in \mathbb{N}, n \geq 4$,

$$
b \leq A\left(m_{n}, m_{n+1}\right)=\left(\frac{r_{m_{n}}}{r_{m_{n+1}}}\right)^{m_{n}} \frac{v\left(r_{m_{n}}\right)}{v\left(r_{m_{n+1}}\right)} \leq b^{9 / 2}
$$

and

$$
b \leq B\left(m_{n}, m_{n+1}\right)=\left(\frac{r_{m_{n+1}}}{r_{m_{n}}}\right)^{m_{n+1}} \frac{v\left(r_{m_{n+1}}\right)}{v\left(r_{m_{n}}\right)} \leq b^{4} .
$$

Proposition 3.2 implies that the numbers $m_{n}$ can chosen to be $n^{2}$ for the weight $v(r)=\exp (-r)$ and $2 n^{2}$ for the weight $v(r)=\exp \left(-r^{2}\right)$. We give the proof of this proposition in several steps.

Lemma 3.3. If $0<m<M$ satisfies $M<2 m$, then

$$
\exp \left(\frac{1}{2} \frac{(M-m)^{2}}{M}\right) \leq\left(\frac{M}{m}\right)^{M} e^{m-M} \leq \exp \left(\frac{(M-m)^{2}}{M}\right) .
$$


Proof. If $0<x<1 / 2$, then

$$
x^{2} / 2 \leq-\log (1-x)-x \leq x^{2} .
$$

This is so since

$$
-\log (1-x)-x=x^{2} / 2+x^{3} / 3+\cdots \geq x^{2} / 2,
$$

and, on the other hand,

$$
x^{2}+\log (1-x)+x=x^{2} / 2-x^{3} / 3-\cdots \geq 0 .
$$

We now set $x:=(M-m) / M$. Clearly $0<x<1 / 2,1-x=m / M$ and $m-M=$ $-M x$. Hence

$$
M(-\log (1-x)-x)=-M \log (m / M)+m-M,
$$

and (3.2) implies $M x^{2} / 2 \leq-M \log (1-x)-M x \leq M x^{2}$, or,

$$
\frac{1}{2} \frac{(M-m)^{2}}{M} \leq M \log (M / m)+m-M \leq \frac{(M-m)^{2}}{M} .
$$

Lemma 3.4. If $0<m<M$ satisfies $M<7 m / 4$, then

$$
\exp \left(\frac{1}{4} \frac{(M-m)^{2}}{m}\right) \leq\left(\frac{m}{M}\right)^{m} e^{M-m} \leq \exp \left(\frac{1}{2} \frac{(M-m)^{2}}{m}\right) .
$$

Proof. We have, for $0<x<3 / 4$,

$$
\log (1+x)-x=-x^{2} / 2+x^{3} / 3-x^{4} / 4+\cdots \geq-x^{2} / 2,
$$

and

$$
-x^{2} / 4-\log (1+x)+x=x^{2} / 4-x^{3} / 3+x^{4} / 4+\cdots \geq x^{2}(1 / 4-x / 3)>0 .
$$

Hence, for these $x$,

$$
-x^{2} / 2 \leq \log (1+x)-x \leq-x^{2} / 4 .
$$

Fix $0<m<M<7 m / 4$. Set $x:=M / m-1$ so that $0<x<3 / 4$. We have

$$
-m(\log (1+x)-x)=m \log (m / M)+M-m,
$$

hence (3.3) implies

$$
\frac{m}{4} x^{2}=\frac{1}{4} \frac{(M-m)^{2}}{m} \leq m \log (m / M)+M-m \leq \frac{1}{2} \frac{(M-m)^{2}}{m}=\frac{m}{2} x^{2} .
$$

Lemma 3.5. Assume that $m_{n}=\alpha n^{2}, n \geq 4$, for some $\alpha>0$. Then

$$
\exp \alpha \leq\left(\frac{m_{n}}{m_{n+1}}\right)^{m_{n}} \exp \left(m_{n+1}-m_{n}\right) \leq \exp (9 \alpha / 4)
$$

and

$$
\exp \alpha \leq\left(\frac{m_{n+1}}{m_{n}}\right)^{m_{n+1}} \exp \left(m_{n}-m_{n+1}\right) \leq \exp (4 \alpha)
$$


Proof. Set, for $n \geq 4, m:=\alpha n^{2}$ and $M:=\alpha(n+1)^{2}$. Then $M-m=\alpha(2 n+1)$ and $M<7 m / 4<2 m$. It is now easy to see that

$$
4 \alpha \leq \frac{(M-m)^{2}}{m} \leq 9 \alpha \text { and } 2 \alpha \leq \frac{(M-m)^{2}}{M} \leq 4 \alpha,
$$

The conclusion follows from Lemmas 3.3 and 3.4.

Proof of Proposition 3.2. In this case the maximum point $r_{m}$ of $r^{m} v(r)$ is $r_{m}=$ $(m / a p)^{1 / p}$, and $v\left(r_{m}\right)=\exp (-m / p)$ for each $m \in \mathbb{N}$. Therefore,

$$
A\left(m_{n}, m_{n+1}\right)^{p}=\left(\frac{m_{n}}{m_{n+1}}\right)^{m_{n}} \exp \left(m_{n+1}-m_{n}\right)
$$

and

$$
B\left(m_{n}, m_{n+1}\right)^{p}=\left(\frac{m_{n+1}}{m_{n}}\right)^{m_{n+1}} \exp \left(m_{n}-m_{n+1}\right) .
$$

We apply Lemma 3.5 for $\alpha:=p(\log b)$ to conclude

$$
b^{p}=\exp (\alpha) \leq A\left(m_{n}, m_{n+1}\right)^{p} \leq \exp (9 \alpha / 4)=b^{9 p / 4},
$$

and

$$
b^{p}=\exp (\alpha) \leq B\left(m_{n}, m_{n+1}\right)^{p} \leq \exp (4 \alpha)=b^{4 p} .
$$

This implies the inequalities in the statement.

Remark 3.6. In view of Lemmas 2.1-2.3, it is of interest to compare the expressions $A(m, n)$ and $B(m, n)$. Let us show here that

$$
\text { (*) } \quad A\left(m_{n}, m_{n+1}\right) \leq B\left(m_{n}, m_{n+1}\right)
$$

for $v(r)=\exp \left(-a r^{p}\right)$ and $m_{n}=\alpha n^{2}, \alpha>0$. It is enough to do the calculation for $m_{n}=n^{2}$. In this case we have

$$
A\left(m_{n}, m_{n+1}\right)=\left(\frac{n}{n+1}\right)^{2 n^{2}} e^{2 n+1} \quad, \quad B\left(m_{n}, m_{n+1}\right)=\left(\frac{n+1}{n}\right)^{2(n+1)^{2}} e^{-2 n-1},
$$

so that $(\star)$ is equivalent to

$$
\frac{\exp (n+1 / 2)}{(1+1 / n)^{n^{2}}} \leq \frac{(1+1 / n)^{(n+1)^{2}}}{\exp (n+1 / 2)}
$$

or

$$
e \leq\left(1+\frac{1}{n}\right)^{\frac{2 n^{2}+2 n+1}{2 n+1}}=: \gamma_{n}
$$

But $\gamma_{n}>(1+1 / n)^{n+1 / 2}=: \eta_{n}$. Thus, (3.4) holds true, since the sequence $\left(\eta_{n}\right)_{n}$ tends to $e$ as $n \rightarrow \infty$, and it is decreasing. To see this last fact, we write

$$
\left(\frac{\eta_{n}}{\eta_{n+1}}\right)^{2}=\frac{(n+1)^{4 n+4}}{n^{2 n+1}(n+2)^{2 n+3}}=\left(\frac{n+1}{n^{\frac{2 n+1}{4 n+4}}(n+2)^{\frac{2 n+3}{4 n+4}}}\right)^{4 n+n}
$$


The logarithm of the expression in the last large parenthesis is of the form

$$
\log x-(r \log (x-1)+(1-r) \log (x+1)),
$$

where $x>1$ and $0<r<1$. This expression is positive, since log is a concave function on $[0, \infty[$. Hence, (3.5) is larger than 1 .

\section{The space of multipliers $\left(H_{v}^{\infty}(\mathbb{C}), \ell^{p}\right)$}

In this final section we show that the above results can be applied to determine some multiplier spaces. Recall that if $A$ and $B$ are vector spaces of complex sequences containing the space of all the sequences with finitely many non-zero coordinates, then the set of multipliers from $A$ into $B$ is

$$
(A, B):=\left\{c=\left(c_{n}\right):\left(c_{n} a_{n}\right) \in B \forall\left(a_{n}\right) \in A\right\} .
$$

Given a strictly increasing, unbounded sequence $J=\left(m_{n}\right)_{n=0}^{\infty} \subset \mathbb{N}$ and $1 \leq$ $p, q \leq \infty$ we denote as in [8], Definition 2,

$$
\ell^{J}(p, q):=\left\{\left(a_{m}\right)_{m=0}^{\infty}:\left(\sum_{m=m_{n}+1}^{m_{n+1}}\left|a_{m}\right|^{p}\right)^{1 / p} \in \ell_{q}\right\}
$$

with the obvious changes when $p$ or $q$ is $\infty$. The space $\ell^{J}(p, q)$ is a Banach space when endowed with the canonically defined norm. Observe that $\ell^{J}(p, p)=\ell_{p}$.

Lemma 4.1. For $1 \leq p \leq \infty$ we have

$$
\left(\ell^{J}(2, \infty), \ell^{p}\right)=\ell^{J}(r, s)
$$

where (a) $r=2 p /(2-p), s=p$, if $1 \leq p<2$; (b) $r=\infty, s=p$, if $2 \leq p<\infty$; and (c) $r=s=\infty$, if $p=\infty$.

Proof. This is a direct consequence of Theorem 23 in [8]. As it is mentioned in that paper, which treats more complicated cases, the proof in the case of our Lemma is similar to that of Theorem 1 in [14].

Theorem 4.2. Let $v$ be a radial weight and let $J=\left(m_{n}\right)_{n=0}^{\infty}$ be a strictly increasing, unbounded sequence of positive integers such that for some $b>2$ and $K \geq b$ we have

$$
\begin{aligned}
b & \leq \min \left(A\left(m_{n}, m_{n+1}\right), B\left(m_{n}, m_{n+1}\right)\right) \\
& \leq \max \left(A\left(m_{n}, m_{n+1}\right), B\left(m_{n}, m_{n+1}\right)\right) \leq K .
\end{aligned}
$$

Let $1 \leq p \leq \infty$. Then $\left(\lambda_{m}\right)_{m=1}^{\infty}$ is a multiplier from $H_{v}^{\infty}(\mathbb{C})$ into $\ell_{p}$ if and only if

$$
\left(\left(\left(v\left(r_{m_{n}}\right) r_{m_{n}}^{m}\right)^{-1}\left|\lambda_{m}\right|\right)_{m=m_{n}+1}^{m_{n+1}}\right)_{n=0}^{\infty} \in \ell^{J}(r, s),
$$


where
(a) $r=2 p /(2-p), s=p$, if $1 \leq p<2$,
(b) $r=\infty, s=p$, if $2 \leq p<\infty$, and
(c) $r=s=\infty$, if $p=\infty$.

Proof. Since $\ell^{p}$ is a solid space, we can apply Lemma 3 in [1] to conclude

$$
\left(H_{v}^{\infty}(\mathbb{C}), \ell_{p}\right)=\left(S\left(H_{v}^{\infty}(\mathbb{C})\right), \ell_{p}\right) .
$$

By Theorem 2.5 it is easy to see that $\left(\lambda_{m}\right)_{m=0}^{\infty} \in\left(S\left(H_{v}^{\infty}(\mathbb{C})\right), \ell_{p}\right)$, if and only if

$$
\left(\left(\left(v\left(r_{m_{n}}\right) r_{m_{n}}^{m}\right)^{-1}\left|\lambda_{m}\right|\right)_{m=m_{n}+1}^{m_{n+1}}\right)_{n=0}^{\infty} \in\left(\ell^{J}(2, \infty), \ell_{p}\right)
$$

The conclusion now follows from Lemma 4.1.

The next corollary is a consequence of Theorem 4.2 and Proposition 3.2.

Corollary 4.3. Let $v(r)=e^{-r}, r \in(0, \infty)$ and $1 \leq p \leq \infty$. Then, the space of multipliers $\left(H_{v}^{\infty}(\mathbb{C}), \ell_{p}\right)$ is the set of sequences $\left(\lambda_{m}\right)_{m=0}^{\infty}$ such that

$$
\left(\sum_{n=1}^{\infty}\left(\sum_{m=n^{2}+1}^{(n+1)^{2}}\left(\left|\lambda_{m}\right| e^{-n^{2}} n^{-2 m}\right)^{\frac{2 p}{2-p}}\right)^{(2-p) / 2}\right)^{1 / p}<\infty,
$$

if $1 \leq p<2$,

$$
\left(\sum_{n=1}^{\infty}\left(\max _{n^{2}<m \leq(n+1)^{2}}\left|\lambda_{m}\right| e^{n^{2}} n^{-2 m}\right)^{p}\right)^{1 / p}<\infty,
$$

if $2 \leq p<\infty$, and

$$
\sup _{n \in \mathbb{N}}\left(\max _{n^{2}<m \leq(n+1)^{2}}\left|\lambda_{m}\right| e^{n^{2}} n^{-2 m}\right)<\infty,
$$

if $p=\infty$.

Acknowledgements. The authors are indebted to the referee for the careful reading of the manuscript and the helpful suggestions.

\section{References}

[1] Anderson, J. M. And Shields, A. L.: Coefficient multipliers of Bloch functions. Trans. Amer. Math. Soc. 224 (1976), no. 2, 255-265.

[2] Bennett, G., Stegenga, D. A. And Timoney R. M.: Coefficients of Bloch and Lipschitz functions. Illinois J. Math. 25 (1981), no. 3, 520-531.

[3] Bierstedt, K.D., Bonet, J. And Galbis, A.: Weighted spaces of holomorphic functions on balanced domains. Michigan Math. J. 40 (1993), no. 2, 271-297. 
[4] Bierstedt, K. D., Bonet, J. and Taskinen, J.: Associated weights and spaces of holomorphic functions. Studia Math. 127 (1998), no. 2, 137-168.

[5] Blasco, Ó.: Multipliers on spaces of analytic functions. Canad. J. Math. 47 (1995), no. $1,44-64$.

[6] Blasco, Ó. And Galbis, A.: On Taylor coefficients of entire functions integrable against exponential weights. Math. Nachr. 223 (2001), 5-21.

[7] Blasco, Ó. And Pavlovic, M.: Coefficient multipliers on Banach spaces of analytic functions. Rev. Mat. Iberoam. 27 (2011), no. 2, 415-447.

[8] Blasco, Ó. and Zaragoza-Benzosa, C.: Multipliers on generalized mixed norm sequence spaces. Abstr. Appl. Anal. 2014, Art. ID 983273, 15 pp.

[9] Bonet, J., Domański, P., Lindström, M. and Taskinen, J.: Composition operators between weighted Banach spaces of analytic functions. J. Austral. Math. Soc. Ser. A 64 (1998), no. 1, 101-118.

[10] Bonet, J. And TAskinen, J.: A note about Volterra operators on weighted Banach spaces of entire functions. Math. Nachr. 288 (2015), no. 11-12, 1216-1225.

[11] Buckley, S. M., Ramanujan, M. S. and Vukotic, D.: Bounded and compact multipliers between Bergman and Hardy spaces. Integral Equations Operator Theory 35 (1999), no. 1, 1-19.

[12] Doubtsov, E.: Characterisations of Hardy growth spaces with doubling weights. Bull. Austral. Math. Soc. 90 (2014), no. 2, 275-282.

[13] Jevtic, M. And Pavlovic, M.: On the solid hull of the Hardy space $H^{p}, 0<p<1$. Michigan Math. J. 54 (2006), no. 2, 439-446.

[14] KellogG, C. N.: An extension of the Hausdorff-Young theorem. Michigan Math. J. 18 (1971), 121-127.

[15] Kislyakov, S. V.: Fourier coefficients of boundary values of functions that are analytic in the disc and bidisc. Spectral theory of functions and operators, II. Trudy Mat. Inst. Steklov. 155 (1981), 77-94, 183-185.

[16] Li, H.: Convergence of Taylor series in Fock spaces. Studia Math. 220 (2014), no. 2, 179-186.

[17] Lusky, W.: On weighted spaces of harmonic and holomorphic functions. J. London Math. Soc. (2) 51 (1995), no. 2, 309-320.

[18] Lusky, W.: On the isomorphism classes of weighted spaces of harmonic and holomorphic functions. Studia Math. 175 (2006), no. 1, 19-40.

[19] Shields, A. L. And Williams, D. L.: Bounded projections, duality and multipliers in spaces of analytic functions. Trans. Amer. Math. Soc. 162 (1971), 287-302.

[20] Tung, J.: Taylor coefficients of functions in Fock spaces. J. Math. Anal. Appl. 318 (2006), no. 2, 397-409.

[21] Vuкотіс, D.: On the coefficient multipliers of Bergman spaces. J. London Math. Soc. (2) 50 (1994), no. 2, 341-348.

The research of Bonet was partially supported by MTM2013-43540-P, GVA Prometeo II/2013/013 and GVA ACOMP/2015/186. The research of Taskinen was partially supported by the Magnus Ehrnrooth and the Väisälä Foundations. 
Received December 14, 2015; revised July 7, 2016.

José Bonet: Instituto Universitario de Matemática Pura y Aplicada IUMPA, Universitat Politècnica de València, E-46071 Valencia, Spain.

E-mail: jbonet@mat.upv.es

Jari Taskinen: Department of Mathematics and Statistics, P.O. Box 68, University of Helsinki, 00014 Helsinki, Finland.

E-mail: jari.taskinen@helsinki.fi 